\title{
Abstracts of Best Poster Awards 6th Annual Health Sciences Poster Day
}

\author{
Faculty of Medicine, Health Sciences Centre, Kuwait University, April 23, 2001
}

\section{Undergraduate Student Award: Job Satisfaction and Stress among Nurses in Kuwait \\ F. Khajah, S. Al-Dessouky, A. Al-Qallaf, A. Al-Yousef, N.M. Shah \\ Department of Community Health and Behavioral Sciences, Kuwait University, Kuwait}

Introduction and Objective: The study sought to assess the degree of reported job satisfaction and stress and examine the association between job stress and job satisfaction among nurses from two general hospitals, namely, Amiri and Adan. Subjects and Methods: Two hundred and thirty nurses from Amiri Hospital and 142 from Adan Hospital, including both medical and surgical wards, answered a self-administered questionnaire. Results: There was no statistically significant difference in the sociodemographic characteristics of nurses in the two hospitals. About $60 \%$ of nurses in each hospital were moderately or very satisfied. Job satisfaction did not differ among male and female nurses in either of the hospitals. About $42 \%$ of nurses in Amiri and nearly 48\% in Adan hospitals were very dissatisfied with their salary. About $31 \%$ of Amiri nurses and 33\% of Adan nurses agreed or strongly agreed that they frequently thought of quitting their job. Amiri nurses had higher (or equal) median scores on all different aspects of satisfaction compared to their counterparts in Adan Hospital. In both hospitals, the average level of stress was significantly associated with educational level. The overall satisfaction and satisfaction with almost all different aspects of the job were significantly and negatively correlated with stress. In general, satisfaction was higher in Amiri Hospital in comparison to Adan Hospital. Conclusion: A substantial percentage of nurses expressed dissatisfaction with salary and other aspects of their job. Factors that negatively affect job satisfaction should be addressed and positive changes must be made to reduce the problem of a nurse shortage in Kuwait.

\section{Postgraduate Student Award: Genetic Polymorphisms Conferring Resistance against HIV-1 Infection and AIDS: Frequency in Kuwait and Genotyping of HIV-1-Positive Kuwaitis}

M.B. Al-Turab, A. Voevodin

Department of Microbiology, Faculty of Medicine, Health Sciences Centre, Kuwait University, Kuwait

Introduction and Objective: The rate of progression of human immunodeficiency virus (HIV) infection to AIDS varies among individuals. The host genetic factors play an important role in long-term nonprogression to AIDS. Most of the genetic polymorphisms conferring resistance against HIV infection and/or delayed progression to AIDS are located in the genes encoding some of the chemokine receptors and chemokines. The most important among them are: CCR5D32, CCR5-m303, CCR5-59029-G/A, CCR2-64I and SDFI-3'A. The objectives of this study are to estimate the frequencies of 59029-G and 59029-A alleles in healthy Kuwaitis, and to genotype HIVinfected Kuwaitis for mutations affecting the pace of progression to AIDS, namely: CCR5-D32, CCR5-m303, 59029-G/A, CCR2-64I and SDFI- $3^{\prime}$ A. Material and Methods: The DNA was extracted from leukocytes of 109 unrelated healthy Kuwaitis and from 27 HIVinfected Kuwaitis by using the 'salting out' method. Genotyping for CCR5-D32, m-303, 59029-G/A, CCR2-64I and SDFI-3'A was performed by PCR/RFLP tests. The fragments obtained after digestion with restriction endonucleases were sized by agarose gel electrophoresis. Results: The frequencies of 59029-G and 59029-A alleles among 109 healthy individuals genotyped were 0.66 (95\% CI 0.59 0.72 ) and 0.34 (95\% CI $0.28-0.41)$, respectively. None of the 27 HIV-infected Kuwaitis carried the resistant alleles for the CCR5D32 and m-303 mutations. The AIDS-resistant genotypes were identified in 2 (homozygosity for SDFI-3'A allele), 9 (heterozygosity for CCR2-64I allele) and 12 (homozygosity for 59029-G allele) patients out of 27 investigated. Nine combinations of genotypes potentially conferring various degrees of resistance to AIDS were identified. Conclusion: The frequency of the AIDS-protective allele 59029-G in healthy Kuwaitis is significantly higher than the frequency of the 59029-A allele and is among the highest reported until now for var-

\begin{tabular}{ll}
\hline KARGER & ( ) 2001 S. Karger AG, Basel \\
Fax +4161306 1234 & 1011-7571/01/0102-0110\$17.50/0 \\
$\begin{array}{l}\text { E-Mail karger@karger.ch } \\
\text { www.karger.com }\end{array}$ & $\begin{array}{l}\text { Accessible online at: } \\
\text { www.karger.com/journals/mpp }\end{array}$
\end{tabular}


ious ethnic groups. Prospective clinical studies are required for establishing the prognostic values of different AIDS-protective genotypes and their combinations in HIV-positive Kuwaitis.

Supported by Kuwait University, Grant No. MI 120 and MSc Research Grant Project from the College of Graduate Studies.

\section{Resident Award: Detection of Antibodies to Extractable Nuclear Antigens by Immunoblotting}

I. Al-Jumah, R. Al-Attiyah, M. Al-Moutieri, S. Abdul-Majeed, A. Malaviya, K. Whaley

Departments of Microbiology and Medicine, Faculty of Medicine, Kuwait University, and Rheumatology Unit, Mubarak Al-Kabeer Hospital, Kuwait

Introduction and Objective: Antibodies to extractable nuclear antigens (ENAs) are autoantibodies which recognize either chromatin-associated antigens or ribonucleoproteins. These autoantibodies occur in connective tissue diseases (CTD), and determining their specificity is of diagnostic value, particularly for undifferentiated CTDs or overlap syndromes, especially mixed CTD (MCTD) Sharpe's syndrome. Previously, using ELISA for ENA antibody detection, we had failed to support the clinical diagnosis of MCTD and there was doubt that it occurred in Kuwaitis. The development of recombinant ENAs has allowed the development of immunoblotting techniques to detect ENA antibodies. The main aim of this study was to evaluate the system for the diagnosis of MCTD. Patients and Methods: Four patients with clinically classical MCTD were the initial target population. ENAs were detected by immunoblotting using the Inno-Lia ANA system (Innogenetics). Nylon membrane strips with bands of bound recombinant ENAs (SmB, RNP70, RNP-A, RNP-C, SS-A/Ro52, SS-A/Ro60, SS-B/La, Cenp-B, Topo-1/Scl-70, Jo1/HRS, ribosomal RNP, histones and poly-dT) were incubated with test sera and bound antibody detected by alkaline phosphataseconjugated antihuman IgG. The strips were then inspected visually or scanned using a Hewlett-Packard scan jet. Results: All 4 patients had anti-RNP-70 and anti-Sm-B (but not anti-Sm-D) antibodies in their sera, which is consistent with MCTD. Conclusion: Our results show that immunoblotting can be used to confirm the diagnosis of MCTD and that MCTD does occur in the Kuwaiti population. We are now evaluating immunoblotting in the diagnosis of a population of patients with undifferentiated CTDs and overlap syndromes.

\section{Basic and Applied Sciences Award: Distribution of Aminoglycoside Modifying Enzymes in Kuwaiti Isolates of High-Level Aminoglycoside-Resistant Enterococci}

\section{E.E. Udo, N. Al-Sweih, P. John, L.E. Jacob, T.D. Chugh}

Department of Microbiology, Faculty of Medicine, Kuwait University, Kuwait

Objective: To determine the distribution of aminoglycoside modifying enzymes (AME) among high-level aminoglycoside-resistant enterococci isolated in some hospitals in Kuwait. Methods:
Antibiotic resistance was determined by the disk diffusion method with disk containing gentamicin $(120 \mu \mathrm{g})$, streptomycin $(300 \mu \mathrm{g})$ and kanamycin $(300 \mu \mathrm{g})$. The minimum inhibitory concentration (MIC) of gentamicin, kanamycin and streptomycin was determined by agar dilution. Genes encoding the three common AME in gram-positive cocci, $\mathrm{AAC}\left(6^{\prime}\right)-\mathrm{APH}\left(2^{\prime \prime}\right), \mathrm{ANT}\left(4^{\prime}\right)$ and $\mathrm{APH}\left(3^{\prime}\right)$, were detected by the polymerase chain reaction. Results: A total of 119 enterococci consisting of 112 E. faecalis, six E. faecium, and one E. casseliflavus were isolated from urines $(54.3 \%)$, wounds $(16.4 \%)$, blood $(10.2 \%)$ and other sources $(18.9 \%)$. The MIC of all three aminoglycosides were $256->400 \mathrm{mg} / \mathrm{l}$. The isolates were also resistant to erythromycin $(98.3 \%)$, ciprofloxacin $(87.4 \%)$, tetracycline $(84.0 \%)$, chloramphenicol $(61.3 \%)$, vancomycin $(5.0 \%)$ and teicoplanin (4.2\%). Genes encoding $\mathrm{AAC}\left(6^{\prime}\right)-\mathrm{APH}\left(2^{\prime \prime}\right)$ were detected in all gentamicin-resistant isolates with $\mathrm{MIC} \geq 256 \mathrm{mg} / \mathrm{l}$. Genes for $\mathrm{APH}\left(3^{\prime}\right)$ were detected in all the streptomycin-resistant isolates with $\mathrm{MIC} \geq 500 \mathrm{mg} / \mathrm{l}$. Genes encoding the ANT $\left(4^{\prime}\right)$ enzyme were not detected. Conclusion: The results of this study established the incidence of AME in aminoglycoside-resistant enterococci isolated in Kuwait.

Supported by Kuwait University, Grant No. MI 111.

\section{Clinical Sciences Award (1): Temporal Sequence of Splenic Dysfunction in Sickle Cell Disease}

A.D. Adekilea , A. Owunwanne b, K. Al-Za'abic, M.Z. Haidera, M. Tulic, S. Al-Mohannadic, S. Gopinath ${ }^{\mathrm{b}}$, M. Mathew ${ }^{\mathrm{b}}$

Departments of apediatrics and ${ }^{\mathrm{b}}$ Nuclear Medicine, Faculty of Medicine, Kuwait University, and 'Department of Nuclear Medicine, Mubarak Al-Kabeer Hospital, Kuwait

Introduction: Previous studies showed that when splenic phagocytic function is impaired, as reflected by nonvisualization of the spleen with ${ }^{99 \mathrm{~m}} \mathrm{Tc}$ colloid scintigraphy, it might be visualized with ${ }^{99 \mathrm{~m}} \mathrm{Tc}$ heat-denatured red blood cells $\left({ }^{99 \mathrm{~m}} \mathrm{TcHdRBC}\right)$. This study was designed to test the hypothesis that in patients with sickle cell disease (SCD), the impairment of splenic phagocytic function occurs first, followed by impairment of the filtration function (tested with

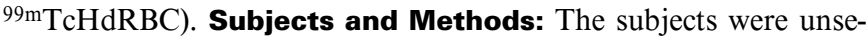
lected SCD patients attending the pediatric hematology clinics in Mubarak Al-Kabeer and Al-Amiri hospitals. All were screened with ${ }^{99 \mathrm{~m}} \mathrm{Tc}$ tin colloid and those whose spleens were partially or not visualized were later imaged with ${ }^{99 m}$ TcHdRBCs. Results: Of the 46 children studied, $18(39.1 \%)$ had good visualization of colloid uptake (group 1), 15 (32.6\%) had partial uptake (group 2) while 13 (28.3\%) had no visualization (group 3 ). When the patients in group 1 were compared to those in the two other groups, the mean $\mathrm{Hb}, \mathrm{MCV}$ and $\mathrm{MCH}$ were significantly higher than in the other two. The prevalence of $\alpha$-thal-2 trait among the SS patients in group 1 was significantly higher than in the other groups $\left(\chi^{2}=85, \mathrm{p}<0.0001\right)$. ${ }^{99 \mathrm{~m} T c H d R B C}$ scintigraphy was carried out on 5 patients in group 2 and the spleen was visible in all while of 12 children in group 3 , the spleen was visible only in 4. Repeat studies in the latter after 1-6 years showed the spleen only in 2. Conclusions: This study confirms that the phagocytic function of the spleen is the first to be impaired while the filtration function, tested by denatured RBC uptake, persists for much longer. Coexistent $\alpha$-thal trait is associated with good spleen function in SS patients. 


\section{Clinical Sciences Award (2): The Correlation between Outdoor Pollens and the Seasonal Variation in Allergic Rhinitis and Asthma in Kuwait}

N. Behbehania, M. Al-Mosawid, N. Arifuodzic ${ }^{d}$ S. Marafiec, L. Ashkananic, M. Moussab, A. Al-Duwaisand

Departments of a Medicine and ${ }^{b}$ Community Medicine, Kuwait University; ${ }^{c}$ Air-Biology Laboratory and dAl-Rashed Allergy Center, Kuwait

Background: Allergic rhinitis (AR) and asthma are common medical problems in Kuwait. Most of the patients who have either AR or asthma are referred to Al-Rashed Allergy Center, the specialized center for allergy in Kuwait. Objectives: To determine if there is a seasonal variation in AR and asthma in Kuwait and correlate the number of new patients referred to the center with the daily pollen count. Methods: The number of new patients per month referred to the center over 3 years (1996-1998) was extracted from the center's records. The daily pollen count from a single monitoring station in Kuwait City was obtained from the Air-Biology Laboratory. Results: There is a significant seasonal variation in the number of new patients with AR referred to the center with a peak in SeptemberOctober of each year (periodic regression analysis $\mathrm{p}<0.001$ ). Although not statistically significant, there are the peaks in the number of new asthma patients referred: March-May and the September-October. The number of new AR patients per month was significantly correlated with two kinds of weed pollens: chenopodium pollens ( $\mathrm{rs}=0.77, \mathrm{p}<0.001$, Spearman rank correlation) and artemisia pollens ( $\mathrm{rs}=0.5, \mathrm{p}=0.002)$, while the number of new asthma patients referred was correlated with grass (gramineae) pollens only ( $\mathrm{rs}=0.42$, $\mathrm{p}=0.01)$. Conclusion: September-October is the main season for seasonal AR in Kuwait and it is mainly due to pollination of certain weeds that exist in Kuwait. The referral pattern for bronchial asthma does not show a statistically significant seasonal variation, but it is correlated with the pollination season of certain grasses in Kuwait. 